\title{
Changes in health-related quality of life in oesophagogastric cancer patients participating in palliative and curative therapies
}

\author{
Mohsen Asadi-Lari ${ }^{1}$, Fatemeh Homaee-Shandiz ${ }^{2}$, Ali Esmaeili-Hesari*3 \\ Received: 11 Jan 2017 \\ Published: 12 Apr 2018
}

\begin{abstract}
Background: Various treatments are used to prolong survival and improve quality of life (QOL). The purpose of this study was to assess the change in QOL scores in patients with Oesophagogastric (OG) cancer undergoing curative intent and palliative therapy.

Methods: This was a mix-designed cohort study with a consecutive sampling of patients with OG cancer who underwent curative or palliative treatment regimens. The QOL, as a determinant of efficacy and impact of cancer care, was evaluated using the European Organization for Research and Treatment of Cancer Quality of Life Questionnaires. QOL data were collected from the eligible subjects at three points of time. The repeated measurement test was used to compare the significance of change in scores.

Results: Overall, 149 patients $(54.4 \%$ male, $61.1 \%$ curative intent, $52.3 \%$ esophageal, $37.6 \%$ gastric, $10.1 \%$ OG junction cancer; with mean age 62 year) with OG cancer were eligible for inclusion in the study. Compared to the palliative group, the curative group was more likely to have an esophageal tumor site, Squamous Cell Carcinoma, and stage 2 (versus stomach, Adenocarcinoma, and stage 4 in the palliative group). In comparing the patients' functional, global health status, and cancer symptom, considering time, group of treatment, and their mutual effect the result indicated significant difference between the intervention groups.

Conclusion: Most patients with Oesophagogastric cancer are diagnosed with an incurable form of the disease. Hence in absence of curative treatment, palliative therapy is the most effective therapy to maintain patient independency and relieve pain and symptom in order to improve their QOL. The present study has shown that palliative similar to curative intervention can improve the QOL in cancer patient especially in short term.
\end{abstract}

Keywords: Oesophagogastric cancer, Health-related quality of life, EORTC QLQ-OG25, EORTC QLQ-C30, Repeated measurement analysis, Iran

Copyright $\subseteq$ Iran University of Medical Sciences

Cite this article as: Asadi-Lari M, Homaee-Shandiz F, Esmaeili-Hesari A. Changes in health-related quality of life in oesophagogastric cancer patients participating in palliative and curative therapies. Med J Islam Repub Iran. 2018(12 Apr);32:31. https://doi.org/10.14196/mjiri.32.31

\section{Introduction}

It has been estimated that in 2012 more than 14.1 million new cancer cases have been identified. Base on the Globocan report there are about 8.2 million cancer deaths and 32.6 million people living with cancer annually. Most of these new cases and deaths have occurred in less developed regions of the world (1). In Iran, after cardiovascular disease and accidents, cancer is the third most common cause of death(2), with a significant rise in incidence rate for the next decades because of population growth, aging, environmental hazards, and change in lifestyle from tradi-

Corresponding author: Ali Esmaeili-Hesari, esmaeilia1@mums.ac.ir

1. Department of Epidemiology and Oncopathology Research Center, Iran University of Medical Sciences, Tehran, Iran.

2. Cancer Research Center, Mashhad University of Medical Sciences, Mashhad, Iran.

3. Social Determinants of Health Research Center, Mashhad University of Medical Sciences, Mashhad, Iran. tional to western one(3-6).

The gastrointestinal tract is the most common organ involved with cancer (7) and the global burden of gastrointestinal tract cancers has been estimated to increase to 8.1 million by 2020 (8). Base on the cancer registry program in Iran, , gastric cancer have the highest frequency in males, followed by breast cancer in females (2). The northeast region of Iran is known to have a highest incidence of Oesophageal cancer (OC) in the world (9). Furthermore, $(\mathrm{OC})$ is a major health problem with increasing

$\uparrow$ What is "already known" in this topic:

Health-related quality of life as a clinical outcome measurement is used to understand and evaluate treatment process as well as the efficacy and impact of cancer care.

$\rightarrow$ What this article adds:

Although HRQL improved over time in patients with oesophagogastric cancer undergone curative intent and palliative therapy, in comparison to patients in the palliative group, those in the curative intent group were more likely to report better scores especially in the long term. 
trend worldwide, making it the eighth most common cancer and the sixth cause of cancer death globally (1). In terms of incidence, GC is the fifth most common cancer and the third leading cause of death in the world (1). Hence, based on these statistics, GC and OC are the most important public health issues and two out of the five most prevalent cancers in Iran $(1,2)$.

The multidisciplinary management and multimodal treatment of OC and GC have been launched to have better outcome in overall patient care, increase curative intent, and longer survival rates $(10,11)$. However, due to the lack of comprehensive screening methods, the diagnosis of OG cancers are usually done when patients are at the advance stages of the disease and when more than two-thirds presented with an unresectable tumor and metastatic disease, which in turn leads to a poor prognosis and a short survival rate (12). Consequently, palliative therapy is the only suitable treatment to prolong survival and quality of life (QOL) in advanced cancer cases (1315). Different cancer treatment methods such as surgery, chemotherapy, radiotherapy, and hormone therapy have shown to have a considerable effect in stopping the progress of tumors, reducing cancer-related pain and discomfort, extending survival rates and in many cases, curing the disease. However, these treatments come with adverse side effects that may last for long or short period of time (16). Therefore, a comprehensive evaluation of new and multimodal treatment is essential in cancer care, as well as creating a base for outcome measurements and assessments of biomedical outcomes of patients. There are many traditional outcome measurements available to determine the efficacy and impact of cancer care and treatment such as operative morbidity, in hospital mortality, tumor response rate, survival time and QOL. Patient-Report Outcomes (PRO) such as the Health-Related Quality of Life (HRQL) as a supplementary outcome to compliment the above measurements is also used to assess cancer care effectiveness and they play an important role in understanding and evaluating the cancer treatment process (17, 18). Recently, the use of HRQL is growing rapidly as a clinical outcome measurement in cancer trials $(16,18)$.

Unfortunately, patients with OG cancer cannot escape various gastrointestinal symptoms and functional disturbances due to the extent of disease or its treatment; therefore, HRQL can be helpful in evaluating the quantity and quality of their treatment. Robust HRQL questionnaires such as the Functional Assessment of Cancer Therapy General (FACT-G) and European Organization for Research and Treatment of Cancer Quality of Life Questionnaires (EORTC QLQ-C30) can assess QOL in patients with cancer (19).

Several studies have compared the QOL of patients who have undergone various procedures for GC and OC treatments $(20,21)$. It is essential to evaluate patients during and after the treatment period due to changes in QOL over time, but no study has longitudinally compared the QOL of patients with OG cancer undergoing curative intent and palliative therapy at certain periods of time. Therefore, the aim of this study was to assess the change in QOL scores in patients with OG cancer during and after curative intent and palliative therapy.

\section{Methods}

\section{Design and participants}

This was a mix-designed cohort study that was conducted in two medical oncology outpatient clinics of two major teaching hospitals. A convenience and purposeful sampling of patients with upper gastrointestinal (GI) cancer were recruited into the study since 2010 to 2013 , from a general and an oncology hospitals located in the city of Mashhad, at northeast of Iran. Before being recruited into the study, subjects were allocated to a curative or palliative regimen based on the treatment protocol of the oncology units. The participants were categorized based on their tumor stage, co-morbidity, global status and their physician's opinion on treatment protocols and medical counseling. Patients were then selected for treatment into two predetermined groups for the study: 1) Curative intent group consisted of patients who were to undergo surgery alone, or neoadjuvant chemotherapy and/or radiotherapy and surgery, adjuvant chemotherapy and/or radiotherapy and surgery. 2) Palliative therapy group, consisted of patients who were to undergo palliative surgery, palliative chemotherapy and/or palliative radiotherapy, and the best supportive available care. Using the patients' medical files who had undergone either curative intent or palliative therapy the QOL data collected longitudinally from the eligible patients, who were placed into one of the two treatment intent groups based on the protocol of the study at three points of time at 2-3 month intervals.

We collected socio-demographic data, which included age, sex, and marital status, type of occupation and place of residency from the participants and also clinical data consisting of tumor site, type, and stage in which patients' records were provided. Severity of the disease was staged, when possible, based on the TNM classification of the American Joint Committee for Cancer (AJCC) staging $(22,23)$.

The inclusion criteria for our study were consisted of patients in various stage of tumor, with at least four months after histopathological diagnosis, under 70 years old, and a pathologic result of squamous cell carcinoma (SCC), adenocarcinoma (ADC) of the esophagus, gastric, or esophagogastric junction (OGJ), based on documented histopathological diagnosis, and an expected survival time of at least six months to participate in our three interview sessions. On the other hand, those with previous or concurrent malignancies, inability to complete and understand the language or content of the questionnaire (Farsi), physical, mental or linguistic impairment, refusal to participate and those who participated in other HRQL study were all excluded.

Of hundred and eighty four subjects took part in the study 35 were excluded from our final analysis due to incomplete data. Moreover 18 subjects at the second interview (four lost to follow up and 14 deaths) and 17 at the third interview (seven lost to follow up and 10 deaths) were excluded. Hence, a total of 149 patients participated in our final analysis. Of the excluded patients, eight patients were from the curative intent group and 27 from the 
palliative therapy group.

\section{Measures}

The QOL was measured using the European Organization for Research and Treatment of Cancer Quality of Life Questionnaire, version 3.0 (EORTC QLQ-C30) and its OG cancer supplementary questionnaire (EORTC QLQOG25) $(24,25)$. The EORTC QLQ-C30 is a self-report multidimensional general cancer specific questionnaire and it is a well-known instrument widely employed internationally to measure QOL in cancer patients. It is an extensively validated instrument with acceptable reliability and validity in different cultures and cancer populations worldwide $(26,27)$, including $\operatorname{Iran}(28)$. The EORTC QLQ-OG25 is used in many different cultures $(24,25)$ and it has been translated and validated into Farsi (29).

All eligible patients were asked to fill in the questionnaires at each interview session after attending the teaching hospital outpatient units for treatment or follow up visits. In addition, our research team designed a sociodemographic questionnaire that asked about sociodemographic data and clinical and pathological features. This information was collected by patient-reported outcome; whereas clinical information was collected from hospital medical records. Patients were asked to fill out the questionnaire by themselves. However, if they had difficulties in filling it out, the questionnaire was completed by face-to-face interviews, where a member of our research team recorded the clinical and histopathological data. The questionnaires were administered at the three interviews, and the first interview considered as the baseline.

\section{Statistical methods}

QOL data for analysis limited to the patients whose in- formation were accessible in the three mentioned time periods. The collected data were analyzed using a descriptive statistical test, the Chi-square test for categorical variables, and the independent t-test to compare numeric variables. The repeated measurement multivariate test was used to examine the significance of changes in QOL scores as a function of observed changes in clinical status in three time periods. All analyses were done using the SPSS software for windows version 18 (PASW Statistic 18 software) (30), and P-values $\leq 0.05$ considered statistically significant.

Institutional Review Board approval for the study was obtained from the Ethic Committee of the Iran University of Medical Sciences (IUMS). Furthermore, written informed consent was obtained from each patient and then the purpose of the study voluntary participation, confidentiality and freedom to leave the study at any time were explained to all of the prospective participants.

\section{Results}

Overall, 149 patients completed the questionnaires in three sessions of interview, which were included in the last analysis. Most of the patients were male (54.4\%), married $(85.9 \%)$, and resided in urban areas $(53.7 \%)$. Mean age of patients was 62 years $(\mathrm{SD}=11)$ ranging between 18-70 years and the majority of patients were 61-70 years old, of whom $52.3 \%$ had esophageal, $37.6 \%$ gastric or OG junction cancers(10.1\%). About $82.6 \%$ underwent TNM staging and most of them classified into stage 3 or 4 (51\%). Histopathological reports showed that 50.3\% (75 cases) had SCC and 52.3\% (78 cases) had an esophageal tumor site. The curative intent group consisted of 91 patients $(61.1 \%)$ and the palliative therapy group consisted of 58 patients $(39.9 \%)$. Included and excluded patients were similar with respect to many features such as sex,

Table1. Demographic and clinical details of patients participated in the study

\begin{tabular}{|c|c|c|c|c|c|c|}
\hline \multirow[t]{2}{*}{ Variable } & \multirow[t]{2}{*}{ Category } & \multicolumn{2}{|c|}{$\begin{array}{l}\text { Curative Treatment } \\
\text { Group }(\mathrm{n}=91)\end{array}$} & \multicolumn{2}{|c|}{$\begin{array}{l}\text { Palliative Treatment } \\
\text { Group }(\mathrm{n}=58)\end{array}$} & \multirow[t]{2}{*}{$\mathrm{p}$} \\
\hline & & No. & $\%$ & No. & $\%$ & \\
\hline Sex & Male & 49 & 53.8 & 32 & 55.2 & 0.875 \\
\hline \multirow[t]{4}{*}{ Age (year) } & Less than 40 & 6 & 6.6 & 5 & 8.6 & 0.807 \\
\hline & $41-50$ & 12 & 13.2 & 9 & 15.5 & \\
\hline & $51-60$ & 25 & 27.5 & 12 & 20.7 & \\
\hline & $61-70$ & 48 & 52.7 & 32 & 55.2 & \\
\hline \multirow[t]{3}{*}{ Marital status } & Married & 77 & 84.6 & 51 & 87.9 & 0.655 \\
\hline & Single & 1 & 1.1 & 0 & 0 & \\
\hline & Separated/divorced/widowed & 13 & 14.3 & 7 & 12.1 & \\
\hline \multirow[t]{4}{*}{ Occupation } & Housekeeper & 40 & 44 & 21 & 36.2 & 0.746 \\
\hline & Employed & 31 & 34 & 21 & 36.2 & \\
\hline & Unemployed & 13 & 14.3 & 9 & 15.5 & \\
\hline & Retired & 7 & 7.7 & 7 & 12.1 & \\
\hline \multirow[t]{3}{*}{ Tumor site } & esophagus & 57 & 62.6 & 21 & 36.2 & 0.008 \\
\hline & Stomach & 26 & 28.6 & 30 & 51.7 & \\
\hline & $\mathrm{O}-\mathrm{G}$ junction & 8 & 8.8 & 7 & 12.1 & \\
\hline \multirow[t]{3}{*}{ Tumor type } & $\mathrm{SCC}$ & 56 & 61.5 & 19 & 32.8 & 0.002 \\
\hline & Adencarcinoma & 34 & 37.4 & 38 & 65.5 & \\
\hline & others & 1 & 1.1 & 1 & 1.7 & \\
\hline \multirow[t]{5}{*}{ Stage of Tumor } & Stage I & 4 & 4.4 & 1 & 1.7 & $<0.001$ \\
\hline & Stage II & 37 & 40.7 & 5 & 8.6 & \\
\hline & Stage III & 31 & 34.0 & 5 & 8.6 & \\
\hline & Stage IV & 1 & 1.1 & 39 & 67.2 & \\
\hline & No staging & 18 & 19.8 & 8 & 13.8 & \\
\hline \multirow{2}{*}{ Residence location } & Urban area & 41 & 45.1 & 39 & 67.2 & 0.007 \\
\hline & Rural area & 50 & 54.9 & 19 & 32.8 & \\
\hline
\end{tabular}


age group, and tumor site, type, and stage; however, both groups had significant statistical differences in respect to type of treatment $(\mathrm{p}<0.001)$ and most of the excluded patients were in the palliative group.

Apart from tumor site, type, and stage, and residency status, there was no significant difference in the other demographic and clinical features between the two groups (curative and palliative). Compared to the palliative group, the curative group significantly had more esophageal tumor site and SCC tumor type versus ADC and GC in the palliative group. Most patients in the curative group were in stage $2(40.7 \%)$ and in the palliative group the patients in stage $4(67.2 \%)$. Socio-demographic and clinical characteristics of the curative and palliative group included in the last analysis are shown in Table 1.

Although the mean score of QOL in all functional scales was higher in the curative in comparison to the palliative group, but the symptom scales and items were opposite at the first interview. However, this difference was statistically significant only in the physical $(p<0.001)$, role $(p=0.007)$, and social $(p=0.049)$ functional scales; in nausea/vomiting $(p=0.014)$, dysphagia $(p<0.001)$, eating restriction $(\mathrm{p}=0.018)$, reflux $(\mathrm{p}=0.007)$, odynophagia $(p=0.017)$, pain and discomfort $(p=0.042)$, and anxiety $(p<0.001)$ symptom scales; and appetite loss $(p=0.005)$, diarrhea $(\mathrm{p}<0.001)$, speaking $(\mathrm{p}=0.005)$, and weight loss $(\mathrm{p}<0.001)$ single items.

Comparison of the patients' functional and global health status during the time, measured by the EORTC QLQ$\mathrm{C} 30$, and the result while considering time, group of treatment, and their mutual effect indicated significant improvement in the two groups. Time was a significant factor in all functional scales and global QOL. In addition, irrespective of time, a significant difference found between the two treatment groups in physical $(\mathrm{p}<0.001)$ and role $(p=0.001)$ functions, and global QOL $(p=0.001)$ (Table 2).

Furthermore, the comparison of the general cancer symptom scales and items showed that the mean score of QOL change in relation to cancer from the time affected indicated a significant change was found in regard to fatigue, nausea or vomiting, the pain scales and the dyspnea, insomnia, and economical single item. However, regarding other aspects such as appetite loss, constipation, and diarrhea time was not a significant factor for change. In general, except for time, there was a significant difference between treatment groups regarding fatigue $(p=0.004)$, nausea or vomiting $(\mathrm{p}=0.002)$, pain $(\mathrm{p}=0.016)$, insomnia $(\mathrm{p}=0.022)$, appetite loss $(\mathrm{p}=0.001)$, and constipation $(\mathrm{p}=0.009)$ (Table 3). Regarding the mutual effect of time

Table 2. Means scores of functional and Global health status scales in treatment intent groups over time (as measured by the EORTC QLQ-C30)

\begin{tabular}{|c|c|c|c|c|c|c|c|}
\hline \multirow[t]{2}{*}{ Functioning scores } & Time & $1^{\text {st }}$ interview & $2^{\text {nd }}$ interview & $3^{\text {rd }}$ interview & & $\mathrm{p}^{*}$ & \\
\hline & Groups & Mean \pm SD & $\mathrm{Mean} \pm \mathrm{SD}$ & $\operatorname{Mean} \pm \mathrm{SD}$ & Time & Group & Time/Group \\
\hline \multirow[t]{2}{*}{ Physical functioning } & Curative & $64 \pm 21$ & $72 \pm 19$ & $75 \pm 23$ & $<0.001$ & $<0.001$ & 0.205 \\
\hline & Palliative & $49 \pm 18$ & $64 \pm 25$ & $61 \pm 28$ & & & \\
\hline \multirow[t]{2}{*}{ Role functioning } & Curative & $70 \pm 24$ & $75 \pm 22$ & $79 \pm 24$ & 0.002 & 0.001 & 0.314 \\
\hline & Palliative & $59 \pm 26$ & $70 \pm 23$ & $66 \pm 27$ & & & \\
\hline \multirow[t]{2}{*}{ Emotional functioning } & Curative & $69 \pm 22$ & $79 \pm 19$ & $82 \pm 18$ & $<0.001$ & 0.073 & 0.402 \\
\hline & Palliative & $68 \pm 24$ & $74 \pm 23$ & $75 \pm 21$ & & & \\
\hline \multirow[t]{2}{*}{ Cognitive functioning } & Curative & $77 \pm 26$ & $92 \pm 16$ & $94 \pm 13$ & $<0.001$ & 0.388 & 0.099 \\
\hline & Palliative & $81 \pm 21$ & $88 \pm 22$ & $90 \pm 16$ & & & \\
\hline \multirow[t]{2}{*}{ Social Functioning } & Curative & $58 \pm 26$ & $65 \pm 27$ & $73 \pm 30$ & $<0.001$ & 0.123 & 0.521 \\
\hline & Palliative & $49 \pm 27$ & $62 \pm 29$ & $69 \pm 28$ & & & \\
\hline \multirow[t]{2}{*}{ Global Health Status } & Curative & $56 \pm 25$ & $63 \pm 23$ & $69 \pm 23$ & 0.001 & 0.001 & 0.332 \\
\hline & Palliative & $48 \pm 24$ & $55 \pm 23$ & $54 \pm 32$ & & & \\
\hline
\end{tabular}

The higher value indicate higher level of functioning and quality of life, min:0, max:100

*- $\mathrm{P}$ value derived from repeated measure ANOVA to examine changes in quality of life

Table 3. Means scores of symptoms scales and single items of life quality in treatment intent groups over time (as measured by the EORTC QLQ$\mathrm{C} 30)$

\begin{tabular}{|c|c|c|c|c|c|c|c|}
\hline \multirow[t]{2}{*}{ Symptom scores } & Time & $1^{\text {st }}$ interview & $2^{\text {nd }}$ interview & $3^{\text {rd }}$ interview & & $\mathrm{p}^{*}$ & \\
\hline & Groups & Mean \pm SD & Mean \pm SD & Mean \pm SD & Time & Group & Time/Group \\
\hline \multirow[t]{2}{*}{ Fatigue } & Curative & $38 \pm 22$ & $34 \pm 20$ & $27 \pm 21$ & $<0.001$ & 0.004 & 0.391 \\
\hline & Palliative & $45 \pm 20$ & $40 \pm 24$ & $39 \pm 26$ & & & \\
\hline \multirow[t]{2}{*}{ Nausea and vomiting } & Curative & $20 \pm 27$ & $18 \pm 20$ & $15 \pm 22$ & 0.036 & 0.002 & 0.725 \\
\hline & Palliative & $31 \pm 28$ & $26 \pm 27$ & $24 \pm 21$ & & & \\
\hline \multirow[t]{2}{*}{ Pain } & Curative & $34 \pm 24$ & $24 \pm 25$ & $20 \pm 23$ & $<0.001$ & 0.016 & 0.941 \\
\hline & Palliative & $41 \pm 22$ & $30 \pm 22$ & $28 \pm 23$ & & & \\
\hline \multirow[t]{2}{*}{ Dyspnea } & Curative & $21 \pm 26$ & $13 \pm 20$ & $13 \pm 23$ & 0.003 & 0.194 & 0.481 \\
\hline & Palliative & $22 \pm 19$ & $15 \pm 21$ & $20 \pm 25$ & & & \\
\hline \multirow[t]{2}{*}{ Insomnia } & Curative & $29 \pm 30$ & $25 \pm 31$ & $19 \pm 26$ & 0.033 & 0.022 & 0.055 \\
\hline & Palliative & $37 \pm 28$ & $27 \pm 29$ & $34 \pm 36$ & & & \\
\hline \multirow[t]{2}{*}{ Appetite loss } & Curative & $32 \pm 31$ & $33 \pm 28$ & $26 \pm 29$ & 0.097 & 0.001 & 0.394 \\
\hline & Palliative & $47 \pm 31$ & $41 \pm 28$ & $40 \pm 35$ & & & \\
\hline \multirow[t]{2}{*}{ Constipation } & Curative & $21 \pm 29$ & $25 \pm 28$ & $18 \pm 27$ & 0.867 & 0.009 & 0.108 \\
\hline & Palliative & $29 \pm 29$ & $28 \pm 33$ & $34 \pm 35$ & & & \\
\hline \multirow[t]{2}{*}{ Diarrhea } & Curative & $3 \pm 11$ & $7 \pm 40$ & $4 \pm 14$ & 0.594 & 0.090 & 0.064 \\
\hline & Palliative & $13 \pm 22$ & $4 \pm 17$ & $9 \pm 25$ & & & \\
\hline \multirow[t]{2}{*}{ Financial difficulties } & Curative & $69 \pm 32$ & $58 \pm 37$ & $51 \pm 39$ & $<0.001$ & 0.376 & 0.700 \\
\hline & Palliative & $71 \pm 33$ & $61 \pm 35$ & $57 \pm 40$ & & & \\
\hline
\end{tabular}

The higher value indicate higher level of functioning and quality of life, min:0, max:100

*- $P$ value derived from repeated measure ANOVA to examine changes in quality of life 
and grouping, no significant difference observed in the functional, and general cancer symptom scales and items, and also the global QOL.

Comparison of the mean score of OG cancer symptom scales and single items over time as measured by the EORTC QLQ-OG25, indicated a decrease in symptom scales in all aspects in the curative group, but in the palliative group these factors varied so that at the first follow up the mean score of symptoms declined and then deteriorated. Statistically, apart from the anxiety scale $(p=0.062)$, there was a significant difference in all OG cancer symptom scales $(p<0.001)$. Moreover apart from odynophagia a significant difference found between the two treatment groups in all mean scores of the symptom scales regardless of time (Table 4).

Nonetheless, comparison of the mean score of OG cancer single symptom items over time, showed a decrease in all single symptom items in the curative group. However, regarding the palliative group, there was a decrease in symptoms in the second interview and an increase in symptoms except in aspects of body image, saliva, and hair loss that indicated an improvement in symptoms. Overall, time was a significant factor for change in the side effect of OG cancer treatment, and consequently, a significant difference detected in eating habit along with sense of taste, body image, saliva, choking sensation, weight loss, and hair loss item. Regardless of time, there was a significant difference between the curative and palliative groups in various aspects such as dry mouth, body image, speech, weight loss, and hair loss (Table 5).

Overall, in comparing the mean score of OG symptom scales and items in the treatment groups, considering the mutual effect of time and grouping, no significant difference was found in symptom scales and items, except in the aspects of weight loss $(p=0.010)$, dysphagia $(p<0.001)$, and odynophagia $(\mathrm{p}=0.047)$. The result showed a steady decrease in these symptoms in the curative group; whereas there was a fluctuation pattern in the palliative group due increased symptom after initial decrease (Tables 4, 5). Comparing these symptom in different interview sessions

Table 4. Means scores of symptoms scales in esophagogastric cancer quality of life questionnaire in treatment intent groups over time (as measured by the EORTC QLQ-OG25)

\begin{tabular}{|c|c|c|c|c|c|c|c|}
\hline \multirow[t]{2}{*}{ Symptom scales* } & \multirow{2}{*}{$\begin{array}{c}\text { Time } \\
\text { Groups }\end{array}$} & \multirow{2}{*}{$\frac{1^{\text {st }} \text { interview }}{\text { Mean } \pm \mathrm{SD}}$} & \multirow{2}{*}{$\frac{2^{\text {nd }} \text { interview }}{\text { Mean } \pm \mathrm{SD}}$} & \multirow{2}{*}{$\frac{3^{\text {rd }} \text { interview }}{\text { Mean } \pm \mathrm{SD}}$} & \multicolumn{3}{|c|}{$\mathrm{p}^{* *}$} \\
\hline & & & & & Time & group & Time/Group \\
\hline \multirow[t]{2}{*}{ OGDYS } & Curative & $28 \pm 19$ & $22 \pm 19$ & $20 \pm 23$ & $<0.001$ & $<0.001$ & $<0.001$ \\
\hline & Palliative & $47 \pm 25$ & $27 \pm 24$ & $31 \pm 35$ & & & \\
\hline \multirow[t]{2}{*}{ OGEAT } & Curative & $33 \pm 21$ & $24 \pm 17$ & $21 \pm 18$ & $<0.001$ & 0.003 & 0.283 \\
\hline & Palliative & $41 \pm 20$ & $29 \pm 21$ & $32 \pm 25$ & & & \\
\hline \multirow[t]{2}{*}{ OGREX } & Curative & $32 \pm 24$ & $27 \pm 22$ & $21 \pm 22$ & $<0.001$ & 0.015 & 0.204 \\
\hline & Palliative & $43 \pm 25$ & $30 \pm 28$ & $34 \pm 26$ & & & \\
\hline \multirow[t]{2}{*}{ OGODYN } & Curative & $27 \pm 22$ & $17 \pm 23$ & $13 \pm 19$ & $<0.001$ & 0.092 & 0.047 \\
\hline & Palliative & $36 \pm 25$ & $15 \pm 20$ & $18 \pm 24$ & & & \\
\hline \multirow[t]{2}{*}{ OGPD } & Curative & $31 \pm 22$ & $26 \pm 26$ & $21 \pm 20$ & $<0.001$ & 0.031 & 0.127 \\
\hline & Palliative & $39 \pm 25$ & $26 \pm 23$ & $31 \pm 25$ & & & \\
\hline \multirow[t]{2}{*}{ OGANX } & Curative & $37 \pm 27$ & $34 \pm 31$ & $33 \pm 35$ & 0.062 & $<0.001$ & 0.087 \\
\hline & Palliative & $59 \pm 28$ & $45 \pm 34$ & $45 \pm 41$ & & & \\
\hline
\end{tabular}

The higher value indicate higher level of functioning and quality of life, min:0, max:100

*-OGDYS, dysphagia; OGEAT, eating restrictions; OGREX, reflux; OGODYN, odynophagia; OGPD, pain and discomfort; OGANX, anxiety;

**-P value derived from repeated measure ANOVA to examine changes in quality of life

Table 5. Means scores of single symptoms item in esophagogastric cancer quality of life questionnaire in treatment intent groups over time (as measured by the EORTC QLQ-OG25)

\begin{tabular}{|c|c|c|c|c|c|c|c|}
\hline \multirow[t]{2}{*}{ Symptom Items* } & Time & $1^{\text {st }}$ interview & $2^{\text {nd }}$ interview & $3^{\text {rd }}$ interview & & $\mathrm{p}^{* *}$ & \\
\hline & Groups & Mean \pm SD & $\mathrm{Mean} \pm \mathrm{SD}$ & Mean \pm SD & Time & Group & Time/Group \\
\hline \multirow[t]{2}{*}{ OGEO } & Curative & $25 \pm 31$ & $16 \pm 22$ & $18 \pm 26$ & 0.007 & 0.160 & 0.412 \\
\hline & Palliative & $28 \pm 33$ & $20 \pm 26$ & $27 \pm 30$ & & & \\
\hline \multirow[t]{2}{*}{ OGDM } & Curative & $38 \pm 29$ & $34 \pm 30$ & $28 \pm 28$ & 0.256 & 0.005 & 0.299 \\
\hline & Palliative & $44 \pm 33$ & $42 \pm 31$ & $44 \pm 31$ & & & \\
\hline \multirow[t]{2}{*}{ OGTA } & Curative & $11 \pm 22$ & $9 \pm 21$ & $5 \pm 17$ & 0.025 & 0.530 & 0.609 \\
\hline & Palliative & $14 \pm 27$ & $8 \pm 14$ & $9 \pm 19$ & & & \\
\hline \multirow[t]{2}{*}{ OGBI } & Curative & $22 \pm 29$ & $12 \pm 21$ & $9 \pm 20$ & $<0.001$ & 0.009 & 0.835 \\
\hline & Palliative & $31 \pm 34$ & $19 \pm 28$ & $16 \pm 21$ & & & \\
\hline \multirow[t]{2}{*}{ OGSV } & Curative & $11 \pm 22$ & $10 \pm 21$ & $6 \pm 18$ & 0.043 & 0.401 & 0.848 \\
\hline & Palliative & $10 \pm 24$ & $6 \pm 19$ & $5 \pm 13$ & & & \\
\hline \multirow[t]{2}{*}{$\mathrm{OGCH}$} & Curative & $16 \pm 24$ & $13 \pm 24$ & $10 \pm 19$ & 0.011 & 0.490 & 0.455 \\
\hline & Palliative & $20 \pm 25$ & $11 \pm 22$ & $13 \pm 23$ & & & \\
\hline \multirow[t]{2}{*}{ OGCO } & Curative & $20 \pm 21$ & $16 \pm 23$ & $14 \pm 22$ & 0.064 & 0.208 & 0.563 \\
\hline & Palliative & $25 \pm 26$ & $17 \pm 25$ & $21 \pm 28$ & & & \\
\hline \multirow[t]{2}{*}{ OGSP } & Curative & $14 \pm 23$ & $10 \pm 19$ & $9 \pm 22$ & 0.181 & 0.013 & 0.071 \\
\hline & Palliative & $21 \pm 24$ & $11 \pm 28$ & $18 \pm 28$ & & & \\
\hline \multirow[t]{2}{*}{ OGWL } & Curative & $16 \pm 26$ & $13 \pm 20$ & $11 \pm 22$ & $<0.001$ & $<0.001$ & 0.010 \\
\hline & Palliative & $39 \pm 32$ & $20 \pm 24$ & $24 \pm 28$ & & & \\
\hline \multirow[t]{2}{*}{ OGHAIR } & Curative & $11 \pm 22$ & $9 \pm 19$ & $8 \pm 23$ & 0.014 & 0.014 & 0.303 \\
\hline & Palliative & $28 \pm 27$ & $21 \pm 31$ & $13 \pm 16$ & & & \\
\hline
\end{tabular}

The higher value indicate higher level of functioning and quality of life, min:0,max:100

*-OGEO, Eating with others; OGDM, Dry mouth; OGTA, sense of taste; OGBI, Body image; OGSV, Saliva; OGCH, Choking; OGCO, Cough; OGSP, speech; OGWL, Weight loss; OGHAIR, Hair loss.

**-P value derived from repeated measure ANOVA to examine changes in quality of life 
indicated a significant difference in mean score of the two treatment groups, except for dysphagia $(p=0.056)$ and weight loss $(p=0.79)$ in the second interview, and odynophagia in the second $(p=0.507)$ and third $(p=0.667)$ interview session.

\section{Discussion}

Although the principal and common end-points in clinical trials are recurrence rate, morbidity, mortality, and survival, but the QOL measurements in patients with cancer are becoming more important and beneficiary. This may be due to the increasing role of patient-based outcome assessments on treatment decision making (31). Hence, the measurement of HRQL in patients with gastric, esophageal and esophagogastric junction cancer is important and should be considered as a part of patient care before, during and after clinical management. Therefore, healthcare providers need to assess QOL routinely by using reliable instruments as early as possible. The EORTC QLQ-C30 and QLQ-OG25 have symptom scales and items relevant to patients undergoing curative intent or palliative therapy that focus on treatment and follow up for OG cancer patients (32).

This mix-designed cohort study consisted of patients with esophagogastric cancer, who were followed by measuring their global QOL, and functional and symptom domains after undergoing curative intent or palliative therapy. Although QOL scores improved over time in both groups, but patients in the curative group reported better QOL than those in the palliative.

This study provided data on the change of HRQL of 149 OG cancer patients undergoing two different treatment regimens, curative or palliative, through nearly four years of follow up using standard QOL measurements. The present study showed that the two treatment groups had a significant difference in regard to tumor site, type and stage. As such, the palliative group had ADC tumor type, gastric cancer and a higher tumor stage. On the other hand, esophagus cancer, SCC tumor type, and lower stages were common in the curative group (Table 1). This result was similar to other studies in that showed SCC in the esophagus (33) and ADC in the stomach are the most common tumors(34), and that carcinoma was associated with poor outcomes (35). Patients with esophageal cancer have earlier disease staging and better survival; whereas, gastric tumors often present with delayed staging and are asymptomatic, and hence most of the patients are diagnosis at advance stages and leaving them with a poor prognosis and low survival rates (36). As the treatment strategy for OG cancer is determined by the stage of the disease and global health status of the patient (37), a potentially curative intent is generally considered in early stages, low grade, and locoregional disease would have a better expected outcome. In contrast, palliative treatment is selected in advanced stages of the disease and in patients with metastatic and unresectable tumors with poor prognosis (38); hence, most of the gastric cancer patients with ADC and those in the advanced stages of their disease had an unresectable disease and so they were under palliative treatment.
Regarding residency status, there was significant difference between the curative and palliative group, so much that most of the palliative group resided in urban areas and most of the curative group in rural areas. Overall, our result showed that about $54 \%$ resided in urban areas, which was different from Amani et al.'s study, in which the majority of gastric cancer patients resided in rural areas(39). Moreover, duration of disease diagnosis was higher for rural residents compared to urban residents; however, residency had no significant effect on delaying diagnosis.

Overall, the repeated measurement multivariate test showed a positive, significant effect regarding the time trend, especially in the curative intent group. By the measured analysis of repeated data we could conclude that participants in the curative intent group in comparison to the palliative group had significantly better and progressive QOL in many functional and symptom aspects. In the following paragraphs we will discuss our findings regarding significant changes in the QOL scores in functional and symptom aspects separately.

At the baseline assessment, patients in the clinically different groups reported a significant difference in baseline HRQL scores in several functional and symptom scales and items so that patients in the curative group had better scores in the functional scale and lower scores in the symptoms scale. Our results are also supported by previous studies (40). There was a significant difference between the functional and symptom domains and items of the HRQL and patients in advanced stages of their disease; and those with poor physical function reported significantly worse functional and symptom scales (32). Also, patients in the curative group reported higher functional scores and lower problems than the palliative group (24).

Our study showed that many aspects of the functional domains of QOL have significantly improved. This is easily understandable because of the study design and the elapse of four months from diagnosis time and the passing of the onset of treatment. It was shown in previous studies that although general aspects of QOL may worsen after treatment, patients have concurrently reported improvements or relief of site-specific issues such as relief of dysphagia. Furthermore; three months after beginning of treatment and early deterioration, functional scales of QOL improved in the first year $(41,42)$. Although the mean score of functional scales improved in the two treatment groups over time, there was no significant difference in emotional and global health status in the palliative group. Korkeila et al. showed that treatment in advance gastri-oesophageal cancer led to the considerable alleviation in eating restrictions and pain, partial improvement in social, emotional and role functions while global health status, physical and cognative functions remainded without change (43). Global health status was worst at the baseline assessment and the mean score of social function had the lowest score in the functional scales. However, the previous studies have shown that the physical and role function scales were the lowest. On the other hand, in Karanicolas et al.'study (44) the measurement of global QOL was the highest and in Kong et al. 
(41) and Kim et al.'s (45) studies global QOL was the worst. Moreover, neoadjuvant chemotherapy had no impact on functional scales, global health status, and symptom scales, but there was significant deference in social functioning (46). Most aspects of QOL were significantly worse than the reference population six months after surgery for OC, and no improvement found at three years, so these patients reported poorer functional scales and more cancer and treatment related symptoms $(47,48)$.

Although improvement in all general cancer and specific OG cancer symptom scores was observed over time in the curative group, a different pattern detected in many symptom aspects of the palliative group so that the mean score of symptoms improved and then deteriorated. However, diarrhea was the only symptom that was worsen and then improved in the curative group. This deterioration can be explained as one of the detrimental adverse effects of treatment in the curative group.

Our findings are supported by the results of $\mathrm{Yu}$ et al., who compared changes of QOL after gastric resection. The authors have found that Physical, Cognitive and Rol functional scores deteriorated significantly in the first year after resection and then showed stability. Although there was no significant change in social functioning, emotional functioning improved constantly during the study period. Nausea, vomiting, fatigue and pain increased in the first after gastrectomy and then decreased steady. (49). Kong et al., who compared changes after gastric cancer surgery, reported that most general cancer symptoms increased in patients at three months and then became steady during the first year without a significant change. Although fatigue, nausea, vomiting, and appetite loss increased at three months and then decreased thereafter, the patients' diarrhea score increased and remained one year after surgery. Regarding gastric cancer symptoms, most of the patients had the highest score at three months postoperation and then decreased afterwards during the first year. However, with respect to dysphagia, no significant difference observed in gastric cancer during the 3-12 month follow ups (41).

A longitudinal cohort study that examined changes of QOL after curative gastric resection indicated that most of the general cancer and stomach cancer symptoms deteriorated at three months and then improved at twelve months (45). In similar studies performed by Shan et al. (50)and Safieddine et al. (51), QOL was evaluated over time after OG cancer surgery. They showed that general symptoms and specific-related $\mathrm{OG}$ cancer symptoms were worse at six weeks to three months post-operation and then improved at 12 months, but diarrhea and reflux remained a problem during the first three years after surgery. Those studies are in agreement with our present results regarding the curative intent group. However, Djarv et al. showed that there was no considerable difference in the mean score of QOL at six months and three years (47).

In agreement with other studies, despite a brief improvement or relief of symptoms in patients in the advanced stages of their disease who underwent palliative care, there was no significant improvement in QOL scores over time and symptoms increased during their follow up period thereafter (52). Moreover, patients with OG cancer in the palliative group experienced more troublesome symptoms than the curative group.

One of the major factors that can describe the impact of curative and palliative therapy for OG cancer on HRQL is to provide a guide for future treatments of patients. Clearly, patients need to have information about the severity of their disease, curing options and treatment-related outcomes, such as impact of treatment on their lifestyle and more importantly expected recovery rates (53). At present, there has not been any documented method to inform patients of their likely HRQL impact based on their curative or palliative therapy.

To improve the QOL of patients with OG cancer, healthcare providers need to give their patients information on temporal problems due to treatment procedures and about possible QOL outcomes that can be expected during the treatment period. For patients with these symptoms, healthcare providers need to develop and provide suitable interventions and special management for each symptom and continuously monitor treatment-related problems.

Our finding may enable healthcare providers to better advise their patients about what to expect during their treatment period and recommend suitable existing treatment choice. This has been the main reason for investigating the QOL of cancer patients, not only during and after their treatment, but also before undergoing multimodal procedures.

Further follow up would be helpful in determining which functional and/or symptom aspects of the scales are permanently damaged and which are just taking a longer time to recover or will deteriorate again. It would also be helpful to study the pattern of QOL change over time, so a patient undergoing curative or palliative therapy can be properly advised regarding QOL and regarding problems encountered due to their treatments.

Unfortunately, one of limitation in this study was lack of baseline data of QOL before treatment in many of the patients because they had already undergone curative care or referred from other centers. Moreover, subjects belonging to this study were recruited only from two major teaching oncology clinics. Despite these limitations, some strengths of our study are that it was a mix-designed and we used internationally validated and standardized instruments (EORTC QLQ-C30 and QLQ-OG25) during our study participants' follow up periods.

Even though the present study was done prospectively and longitudinally with a high acceptance rate using validated multi-dimensional, disease-specific tools, the sample size was small. Chronological changes of QOL may differ according to the type of procedure performed in the curative and palliative group separately. Nevertheless in this study, there were not enough cases for each of the therapy groups. Therefore, further study comparing the type of therapy in the curative and palliative groups is necessary.

\section{Conclusion}

Although intent to treatment was related to many fac- 
tors, the result from this prospective, longitudinal study indicated that the curative care procedures for OG cancer had a short time, and negative impact on most aspects of self-reported HRQL that usually improved within the first year after start of treatment, while in the palliative therapy group, had an improvement in QOL scores for a short time which then deteriorated. Therefore, these results may be used to inform patients regarding what are to be expected during and after treatment and to manage the disease timely, and provide supportive interventions. Furthermore, future studies are required to develop aids for communicating HRQL results to healthcare providers and also patients enjoy a better understanding of the treatment and management of cancer within a clinical context.

\section{Acknowledgements}

The authors greatly appreciate all the patients who participated in this study and the personal of the oncology clinics of the teaching hospitals of the Mashhad University of Medical Sciences.

\section{Conflict of Interests}

The authors declare that they have no competing interests.

\section{References}

1. Ferlay J, Soerjomataram I, Dikshit R, Eser S, Mathers C, Rebelo M, et al. Cancer incidence and mortality worldwide: sources, methods and major patterns in GLOBOCAN 2012. Int $\mathrm{J}$ Cancer. 2015;136(5):E359-86.

2. Radmard AR. Five common cancers in Iran. Arch.Iran Med. 2010;13(2):143.

3. Olefson S, Moss SF. Obesity and related risk factors in gastric cardia adenocarcinoma. Gastric cancer; Gastric cancer. 2015;18(1):23-32.

4. Denholm R, Schuz J, Straif K, Ali FM, Bonas F, Gjebrea O, et al. Environmental carcinogen exposure and lifestyle factors affecting cancer risk in Qatar: findings from a qualitative review. EMHJEastern Mediterranean health journal. 2016;22(3):219-27.

5. Chen Y, Yu C, Li Y. Physical activity and risks of esophageal and gastric cancers: a meta-analysis. PloS one. 2014;9(2):e88082.

6. White MC, Holman DM, Boehm JE, Peipins LA, Grossman M, Henley SJ. Age and cancer risk: a potentially modifiable relationship. Am J Pre Med. 2014 Mar;46(3 Suppl 1):S7-15.

7. Pourhoseingholi MA, Fazeli Z, Ashtari S, Bavand-Pour FS. Mortality trends of gastrointestinal cancers in Iranian population. Gastroenterol Hepatol Bed Bench. 2013;6(Suppl 1):S52-7.

8. Pourhoseingholi MA, Vahedi M, Baghestani AR. Burden of gastrointestinal cancer in Asia; an overview. Gastroenterol Hepatol Bed Bench. 2015;8(1):19-27.

9. Golalipour G, Semnani S, Safaie B, Rajaie S, Sedaghat SM, Kamalinia HR, et al. Predictors of survival in oesophageal cancer patients in a high-risk area in Northern Iran: the role of health services utilisation. Eur J Cancer Care (Engl). 2017 Jan;26(1).

10. Boniface MM, Wani SB, Schefter TE, Koo PJ, Meguid C, Leong S, et al. Multidisciplinary management for esophageal and gastric cancer. Cancer Manag Res. 2016 Apr;8:39-44.

11. Meguid C, Schulick RD, Schefter TE, Lieu CH, Boniface M, Williams $\mathrm{N}$, et al. The Multidisciplinary Approach to GI Cancer Results in Change of Diagnosis and Management of Patients. Multidisciplinary Care Impacts Diagnosis and Management of Patients. Ann Surg Oncol. 2016 Nov; 23(12): 3986-3990.

12. Dittmar Y, Rauchfuss F, Goetz M, Jandt K, Scheuerlein H, Heise M, et al. Non-curative gastric resection for patients with stage 4 gastric cancer--a single center experience and current review of literature. Langenbeck's Arch Surg. 2012;397(5):745-53.

13. Sun J, Song Y, Wang Z, Chen X, Gao P, Xu Y, et al. Clinical significance of palliative gastrectomy on the survival of patients with incurable advanced gastric cancer: a systematic review and meta- analysis. BMC cancer. 2013;13(1):577.

14. Liu Y, Feng Y, Gao Y, Hou R. Clinical benefits of combined chemotherapy with S-1, oxaliplatin, and docetaxel in advanced gastric cancer patients with palliative surgery. OncoTargets Ther. 2016;9:1269-73.

15. Sun V, Krouse RS. Palliative surgery: incidence and outcomes. Semin Oncol Nurs. 2014;30(4):234-41.

16. Numico G, Longo V, Courthod G, Silvestris N. Cancer survivorship: long-term side-effects of anticancer treatments of gastrointestinal cancer. Curr Opin Oncol. 2015;27(4):351-7.

17. Ferrans CE. Advances in measuring quality-of-life outcomes in cancer care. Semin Oncol Nurs. 2010;26(1):2-11.

18. Kenne Sarenmalm E, Oden A, Ohlen J, Gaston-Johansson F, Holmberg SB. Changes in health-related quality of life may predict recurrent breast cancer. Eur J Oncol Nurs. 2009;13(5):323-9.

19. King MT, Bell ML, Costa D, Butow P, Oh B. The Quality of Life Questionnaire Core 30 (QLQ-C30) and Functional Assessment of Cancer-General (FACT-G) differ in responsiveness, relative efficiency, and therefore required sample size. J Clin Epidemiol. 2014;67(1):100-7.

20. Chung HW, Chien LY, Huang SM, Tai CJ, Tai CJ. Changes in symptom patterns and health-related quality of life of cancer patients before and after chemotherapy. J Tradit Chin Med. 2016;36(3):32631.

21. Park S, Chung HY, Lee SS, Kwon O, Yu W. Serial comparisons of quality of life after distal subtotal or total gastrectomy: what are the rational approaches for quality of life management? J Gastric Cancer. 2014;14(1):32-8

22. Rice TW, Blackstone EH, Rusch VW. 7th edition of the AJCC Cancer Staging Manual: esophagus and esophagogastric junction. Ann Surg Oncol. 2010;17(7):1721-4.

23. Washington K. 7th edition of the AJCC cancer staging manual: stomach. Ann Surg Oncol. 2010;17(12):3077-9.

24. Onate-Ocana LF, Velazquez-Monroy N, Vazquez L, EspinosaMireles-de-Villafranca P, Nunez-Rosas E, Ovando-Lezama M, et al. Clinical validation of the EORTC QLQ-OG25 questionnaire for the evaluation of health-related quality of life in Mexican patients with esophagogastric cancers. Psychooncology. 2012;21(7):745-53.

25. Tomaszewski KA, Puskulluoglu M, Biesiada K, Bochenek J, Nieckula J, Krzemieniecki K. Validation of the polish version of the eortc QLQ-C30 and the QLQ-OG25 for the assessment of healthrelated quality of life in patients with esophagi-gastric cancer. J Psychosoc Oncol. 2013;31(2):191-203.

26. Ayana BA, Negash S, Yusuf L, Tigeneh W, Haile D. Reliability and Validity of Amharic Version of EORTC QLQ-C 30 Questionnaire among Gynecological Cancer Patients in Ethiopia. PloS one. 2016;11(6):e0157359.

27. Magaji BA, Moy FM, Roslani AC, Law CW, Sagap I. Psychometric Validation of the Malaysian Chinese Version of the EORTC QLQC30 in Colorectal Cancer Patients. Asian Pac J Cancer Prev. 2015;16(18):8107-12.

28. Safaee A, Moghim Dehkordi B. Validation study of a quality of life (QOL) questionnaire for use in Iran. Asian Pac J Cancer Prev. 2007;8(4):543-6.

29. Hesari AE, Lari MA, Shandiz FH. Psychometric Analysis of a Persian Version of the European Organization for Research and Treatment of Cancer OG25 Quality of Life Questionnaire in Oesophagogastric Cancer Patients. Asian Pac J Cancer Prev. 2014;15(6):2739-45.

30. Norusis MJ, Inc. S. PASW Statistics 18 Guide to Data Analysis: Prentice Hall Press; 2010. 672 p.

31. Nelson EC, Eftimovska E, Lind C, Hager A, Wasson JH, Lindblad S. Patient reported outcome measures in practice. BMJ. 2015;350:g7818.

32. Lagergren P, Fayers P, Conroy T, Stein HJ, Sezer O, Hardwick R, et al. Clinical and psychometric validation of a questionnaire module, the EORTC QLQ-OG25, to assess health-related quality of life in patients with cancer of the oesophagus, the oesophago-gastric junction and the stomach. Eur J Cancer. 2007;43(14):2066-73.

33. Arnold M, Soerjomataram I, Ferlay J, Forman D. Global incidence of oesophageal cancer by histological subtype in 2012. Gut. 2015;64(3):381-7.

34. Almasi Z, Rafiemanesh H, Salehiniya H. Epidemiology characteristics and trends of incidence and morphology of stomach cancer in Iran. Asian Pac J Cancer Prev. 2015;16(7):2757-61. 
35. Moorcraft SY, Fontana E, Cunningham D, Peckitt C, Waddell T, Smyth EC, et al. Characterising timing and pattern of relapse following surgery for localised oesophagogastric adenocarcinoma: a retrospective study. BMC Cancer. 2016;16:112.

36. Sitarz R, Skierucha M, Mielko J, Offerhaus GJA, Maciejewski R, Polkowski WP. Gastric cancer: epidemiology, prevention, classification, and treatment. Cancer Manag Res. 2018;10:239-48.

37. Lordick F, Mariette C, Haustermans K, Obermannova R, Arnold D. Oesophageal cancer: ESMO Clinical Practice Guidelines for diagnosis, treatment and follow-up. Ann Oncol. 2016;27(suppl 5):v50-v7.

38. Groene O, Crosby T, Hardwick RH, Riley S, Greenaway K, Cromwell D. A population-based observational study on the factors associated with the completion of palliative chemotherapy among patients with oesophagogastric cancer. BMJ open. 2015;5(3): $\mathrm{e} 006724$

39. Amani F, Sadrkabir M, Ahari SS, Barzghari S, Yazdanbod A, Sabzevari A, et al. Epidemiology of Gastric Cancer in Northwest Iran: 2003-2011. Middle East Journal of Cancer. 2015;6(3):189-93.

40. Sadighi S, Montazeri A, Sedighi Z, Mohagheghi MA, Froutan H. Quality of life in patients with gastric cancer: translation and psychometric evaluation of the Iranian version of EORTC QLQSTO22. BMC Cancer. 2009;9:305.

41. Kong $\mathrm{H}, \mathrm{Kwon} \mathrm{OK}, \mathrm{Yu} \mathrm{W}$. Changes of quality of life after gastric cancer surgery. J Gastric Cancer. 2012;12(3):194-200.

42. Ramakrishnaiah V, Dash NR, Pal S, Sahni P, Kanti CT. Quality of life after oesophagectomy in patients with carcinoma of oesophagus: A prospective study. Indian J Cancer. 2014;51(3):346-51.

43. Korkeila EA, Salminen T, Kallio R, Mikkola M, Auvinen P, Pyrhonen S, et al. Quality of life with biweekly docetaxel and capecitabine in advanced gastro-oesophageal cancer. Support Care Cancer. 2017;25(9):2771-7.

44. Karanicolas PJ, Graham D, Gönen M, Strong VE, Brennan MF, Coit DG. Quality of Life After Gastrectomy for Adenocarcinoma: A Prospective Cohort Study. Ann Surg. 2013;257(6):1039-46.

45. Kim AR, Cho J, Hsu YJ, Choi MG, Noh JH, Sohn TS, et al. Changes of quality of life in gastric cancer patients after curative resection: a longitudinal cohort study in Korea. Ann Surg. 2012;256(6):1008-13

46. Tatematsu N, Ezoe Y, Tanaka E, Muto M, Sakai Y, Tsuboyama T. Impact of neoadjuvant chemotherapy on physical fitness, physical activity, and health-related quality of life of patients with resectable esophageal cancer. Am J Clin Oncol. 2013;36(1):53-6.

47. Djarv T, Lagergren J, Blazeby JM, Lagergren P. Long-term healthrelated quality of life following surgery for oesophageal cancer. $\mathrm{Br} \mathrm{J}$ Surg. 2008;95(9):1121-6.

48. Fuchs H, Holscher AH, Leers J, Bludau M, Brinkmann S, Schroder $\mathrm{W}$, et al. Long-term quality of life after surgery for adenocarcinoma of the esophagogastric junction: extended gastrectomy or transthoracic esophagectomy? Gastric cancer. 2016;19(1):312-7.

49. Yu W, Park KB, Chung HY, Kwon OK, Lee SS. Chronological Changes of Quality of Life in Long-Term Survivors after Gastrectomy for Gastric Cancer. Cancer Res Treat. 2016;48(3):10306.

50. Shan B, Shan L, Morris D, Golani S, Saxena A. Systematic review on quality of life outcomes after gastrectomy for gastric carcinoma. J Gastrointest Oncol. 2015;6(5):544-60.

51. Safieddine N, Xu W, Quadri SM, Knox JJ, Hornby J, Sulman J, et al. Health-related quality of life in esophageal cancer: effect of neoadjuvant chemoradiotherapy followed by surgical intervention. J Thorac Cardiovasc Surg. 2009:137(1):36-42.

52. Al-Batran SE, Ajani JA. Impact of chemotherapy on quality of life in patients with metastatic esophagogastric cancer. Cancer. 2010;116(11):2511-8.

53. King J, Chamberland P, Rawji A, Ager A, Leger R, Michaels R, et al. Patient educational needs of patients undergoing surgery for lung cancer. Journal of cancer education. J Cancer Educ. 2014;29(4):8027. 